\title{
Gestão da informação utilizando o método infomapping
}

Marta Lígia Pomim Valentim

Doutora em Ciências da Comunicação pela Escola de Comunicações e Artes da Universidade de São Paulo (ECA/USP) em 200;. Mestre pela PUC-Campinas em 1995. Docente de graduação e pósgraduação Lato e Stricto Sensu da Universidade Estadual Paulista (UNESP/ Marília); Pesquisadora do CNPq na área de inteligência competitiva organizacional; Coordena o projeto de pesquisa 'Inteligência Competitiva Organizacional'. Organizadora e uma das autoras do livro "Métodos Qualitativos de Pesquisa em Ciência da Informação"; Organizadora e uma das autoras do livro; Exerce o cargo de Vice-Presidente da Asociación de Educación e Investigación en Bibliotecología, Archivología, Ciencias de la Información y Documentación de Iberoamérica y ElCaribe.

Elisabeth Leão de Carvalho

Mestre em Ciência da Informação pela Pontifícia Universidade Católica de Campinas (PUCCAMP), em 2001; Especialista em Gestão de Projetos pela Universidade Estadual de Londrina (UEL), curso interinstitucional com a Université Des Sciences et Tecnologies de Lille, França, em2002; Especialista em Gerência de Unidades de Informação em Ciência e Tecnologia pela UEL, em 1997; Docente de graduação do Departamento de Ciência da Informação, no período de 2003 a 2005. Bibliotecária; Chefe da Divisão de Sistemas Micrográficos da Assessoria de Tecnologia e Informática (ATI) da UEL, no período de 1981 a 2005; Atualmente atua na Divisão de Apoio 
Tecnológico do Sistema de Arquivos (SAUEL) da UEL.

\section{Luana Maia Woida}

Mestranda em Ciência da Informação pela Universidade Estadual Paulista (UNESP/ Marilia); Bacharel em Administração pela Universidade Estadual de Londrina (UEL), em 2005; Docente substituto, em nível de graduação da Fundação Universidade Federal de Rio Grande (FURG-RS), em 2006.

Elisete Lopes Cassiano

Especialista em Gerência de Unidades de Informação, pela Universidade Estadual de Londrina (UEL).

A gestão da informação é extremamente importante para as organizações. O mapeamento informacional é um instrumento que pode ser utilizado no âmbito da gestão da informação. Para realizar o mapeamento informacional, pode-se utilizar o método denominado "Infomapping", criado por Burk Jr. e Horton Jr., que se constitui em uma valiosa ferramenta para gerenciar os recursos de informação de uma organização. Ele permite descobrir com exatidão o grau de desconhecimento que se tem sobre as fontes, serviços e sistemas com os quais se desenvolve o trabalho informacional da organização. $O$ processo consiste em criar um levantamento de todas as informações, que supostamente constituem-se num recurso informacional gerado internamente ou produzido externamente, que impactam diretamente as atividades corporativas.

Palavras-Chave: Gestão da Informação; Fluxos de Informação; Fluxos Formais; Infomapping.

\section{Information management using the infomapping method}

Information management is extremely important for organizations. Information mapping (Infomapping) is an 
instrument that can be utilized in the context of information management. To perform information mapping, a method created by Burk Jr. and Horton Jr. "Infomapping" - can be utilized which is a valuable tool for managing information resources of an organization. The method allows to accurately uncover the degree of unfamiliarity that one has about the resources, service and systems with which the work of organization information is done. The process consists of surveying all information, that are supposedly an information resource -either internally generated or externally produced - and that directly influences the corporate activities.

Keywords: Information Management; Information Flows; Formal Flows; Infomapping.

Recebido em 13.12.2007 Aceito em 06.03.2008

\section{Introdução}

Toda organização necessita de informação para o desempenho de suas atividades. A informação, nos últimos anos, constituiu-se em um capital tão vital quanto os demais ativos tangíveis. Como qualquer outro recurso, a informação deve ser reconhecida por sua importância na estrutura organizacional, visto que é insumo básico para o desenvolvimento das diversas atividades estratégicas, táticas e operacionais.

[...] é importante compreender a organização como núcleo da sociedade, no sentido, de que ela congrega pessoas, sustenta a economia, gera empregos, profissionaliza e especializa a atuação dos indivíduos, em suma, influencia a cultura e a própria sociedade (VALENTIM, 2007, p. 09).

As funções de uma organização estão, ou deveriam estar, direta ou indiretamente ligadas a sua missão e seus objetivos, pois todos os indivíduos que compõem seu quadro corporativo necessitam de informações para subsidiar suas atividades, desde a resolução de pequenos problemas até a tomada de decisão de grande impacto. As informações necessárias para a realização desses fluxos e processos corporativos, tanto administrativos quanto técnicos, podem estar dentro ou fora do ambiente de trabalho, assim como podem ou não ser registradas em diferentes suportes.

Os vários setores de uma organização produzem informações e conhecimento, acumulando assim, um grande volume de informação. Muitas organizações não possuem uma política informacional que permita a gestão, o processamento e a disseminação da informação de forma eficaz. 
Além do cenário acima mencionado, não se deve esquecer o papel econômico-social que as organizações têm junto à sociedade. Ou seja, elas têm um papel primordial, por serem responsáveis pela geração de riqueza, bem como pela qualidade da condição humana, uma vez que propiciam condições de subsistência sob vários enfoques: alimentação, moradia, educação, saúde entre outros.

Nesse sentido, gerenciar informações requer, primeiramente, uma cultura voltada à informação, uma administração participativa e, também, o comprometimento de cada indivíduo no processo. Além disso, o compartilhamento de ações e responsabilidades fará com que esse gerenciamento aconteça de forma eficiente e eficaz.

Entende-se a gestão da informação como um conjunto de ações que visa desde a identificação das necessidades informacionais, o mapeamento dos fluxos formais (conhecimento explícito) de informação nos diferentes ambientes da organização, até a coleta, filtragem, análise, organização, armazenagem e disseminação, objetivando apoiar o desenvolvimento das atividades cotidianas e a tomada de decisão no ambiente corporativo. A gestão da informação, portanto, deve se preocupar com os documentos gerados, recebidos e utilizados para as atividades do negócio corporativo. A gestão documental ou gestão de documentos faz parte desse processo.

A tomada de decisão, ação individual ou coletiva, também necessita de dados, informação e conhecimento alicerçando a ação. Isso significa que cada decisão poderá influenciar sobremaneira o rumo da organização. Portanto, dados e informações direcionados ao processo decisório devem ser foco da gestão da informação.

Essa massa informacional, composta por diferentes tipologias e fontes de informação deve, necessariamente, ser prospectada e monitorada, filtrada, organizada, analisada, disseminada para os indivíduos da organização, pois o sistema será o grande alimentador da gestão da informação.

- Para Ponjuán Dante (1998, p.135) a gestão da informação visa:

- Maximizar o valor e os benefícios derivados do uso da informação;

- Minimizar o custo de aquisição, processamento e uso da informação;

- Determinar responsabilidades para o uso efetivo, eficiente e econômico da informação;

- Assegurar um fornecimento contínuo da informação.

A gestão da informação exige certo investimento por parte das organizações, assim como exige tempo e capacitação de pessoas para a sua implantação. Para uma organização que pretende adotar um modelo estratégico de gestão da informação, o planejamento e a informação são elementos essenciais. O planejamento porque estabelece as metas, os 
objetivos, as etapas e as fases que devem ser realizadas; a informação é essencial para a formulação, o controle e a retroalimentação das estratégias organizacionais (CASTRO; LIMA; CARVALHO, 1999, p.19).

A organização é entendida, portanto, a partir do enfoque sistêmico, de que é uma totalidade integrada através de diferentes níveis de relações; sua natureza é dinâmica e suas estruturas não são rígidas, mas sim flexíveis, embora estáveis. Ela é resultante das interações e da interdependência de suas partes (CAPRA, 2002). O enfoque sistêmico tem sido empregado de diversas formas, sendo um importante instrumento de gestão.

A idéia de prospecção de demandas pode ser feita dentro de um enfoque disciplinar ou de um enfoque sistêmico. No primeiro caso, consideram-se as necessidades atuais e futuras de informação e conhecimento (CASTRO; LIMA; CARVALHO, 1999,).

Os elementos principais que devem obrigatoriamente participar desse tipo de gestão são:

a) Elementos operativos: hardware, software, base de dados, procedimentos e operadores;

b) Elementos de apoio à decisão: rotinas de decisão programadas e não programadas;

c) Atividades gerenciais: planejamentos estratégico, tático e operacional e seus acompanhamentos e avaliações;

d) Funções organizacionais: produção, mercado, serviços, pessoal, contabilidade e finanças, administração, processamento da informação.

Para desenvolver um sistema de gestão da informação, os elementos constituintes devem ser programados de forma a coletar, processar e oferecer informações para a tomada de decisão gerencial (CASTRO; LIMA; CARVALHO, 1999).

É importante que a gestão da informação envolva todos os setores de uma determinada organização como, por exemplo, os setores que produzem, recebem, utilizam e disseminam informações no espaço corporativo.

Para isso, pode-se utilizar inicialmente um método de mapeamento denominado Infomapping. Este método foi criado por Cornelius Burk Jr. e Forest Horton Jr. e constitui-se numa valiosa ferramenta para gerenciar os recursos informacionais de uma organização, quer pública ou privada.

Existe a possibilidade de se estabelecer fluxos escalonados e hierarquizados de informação, de forma que a tomada de decisão seja coerentemente fracionada nos diversos escalões gerenciais, o que torna possível que a informação gerada em cada nível seja processada e utilizada para a gestão em vários níveis organizacionais, conforme modelo a seguir: 


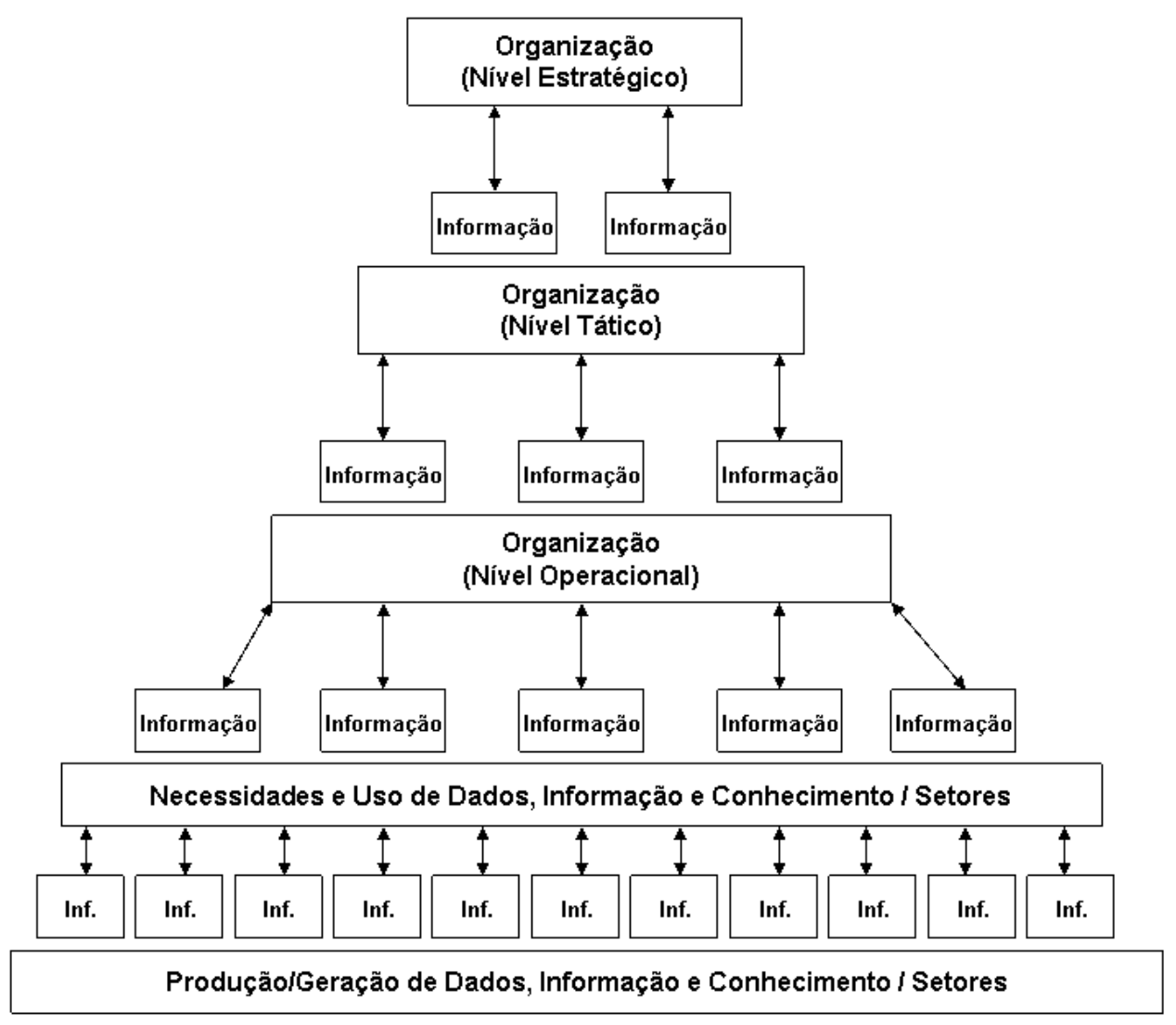

Figura 1 - Fluxos de Informação Hierarquizados.

Fonte - Adaptado de Bolivar et al (1997).

\section{Método infomapping}

O método infomapping criado por Burk Jr. e Horton Jr., em 1988, constitui-se numa valiosa ferramenta para gerenciar os recursos de informação de uma organização. Permite descobrir com exatidão o grau de desconhecimento que se tem sobre as fontes, serviços e sistemas não tradicionais com os quais se desenvolve o trabalho informacional da organização. O processo consiste em criar um levantamento de todas as informações, que supostamente constituem-se num recurso gerado internamente ou produzido externamente.

Para Burk Jr. e Horton Jr. (1988), a informação pode ser gerenciada como um recurso informacional, porém é importante observar quais são realmente as informações relevantes para a organização, pois nem toda informação pode ser considerada um recurso.

Segundo esses autores, o mapeamento indicará os problemas e apresentará as oportunidades, bem como as forças e fraquezas relativas à qualidade da informação, acessibilidade, desempenho, uso e efetividade, valor real e desperdício de investimento, causado pelo uso inadequado da informação.

Em suma, quando o mapeamento dos recursos de informação for inteiramente processado, a organização terá a possibilidade de fazer a 
avaliação de suas fontes, serviços e sistemas, bem como de identificar os meios mais indicados para controlar e acessar as informações disponíveis.

Compreender que a informação é um recurso corporativo importante é fundamental. Os autores propõem o conceito de "Entidade de Recursos de Informação", que é caracterizado pelo conjunto: pessoas, coisas, energia, informação e outros elementos que têm a capacidade de criar, adquirir, prover, processar, armazenar ou disseminar informação.

Burk Jr. e Horton Jr. (1988) ainda enfatizam um princípio determinante para a elaboração do mapeamento de informação, que é o de poder contar com o consentimento e o compromisso da instância máxima da organização, uma vez que esse processo requer tempo, exige determinados recursos e, assim, o apoio por parte deste nível é imprescindível.

O método pode ser estruturado em quatro etapas básicas: Mapeamento preliminar; Determinação de custos e atribuição de valor; Preparo das técnicas do mapeamento da informação; Identificação dos recursos da organização.

$1^{\text {a }}$ Etapa - Mapeamento Preliminar: consiste em desenvolver um conjunto de ações com vistas a identificar de forma preliminar as fontes, serviços, produtos e sistemas que sejam considerados recursos informacionais relevantes para a organização. Um processo importante nessa fase é o estudo da documentação gerada na organização, bem como outras medidas que permitam conhecer a organização e seus líderes

$2^{a}$ Etapa - Determinação de Custos e Atribuição de Valor: nessa etapa o objetivo é recolher um conjunto de elementos relativos aos recursos de informação identificados na primeira etapa. Entre eles, o custo de cada uma das entidades de recursos de informação relevantes para a organização, às quais se atribue um determinado valor. Em seguida, se ordena as informações identificadas segundo dois critérios:

- Custos: consiste em avaliar os custos do acesso às informações, como, por exemplo, bancos e bases de dados (criação, manutenção e atualização), remuneração do profissional responsável, material de escritório, entre outros. Isso se faz de acordo com quatro categorias: alto custo, médio custo, baixo custo e custo mínimo ou nulo.

- Atribuição de Valores: consiste em agregar valor às informações. Fundamenta-se na transferência de informação como resposta intensiva a um processo humano, tanto nas atividades formalizadas, chamadas de sistemas, quanto nas atividades não formalizadas. O enfoque deste critério está na relação entre os sistemas e os usuários, utilizando cinco categorias, a saber: qualidade da informação em si, utilidade da informação, impacto na produtividade organizacional, impacto na eficácia organizacional e impacto na posição financeira. 
$3^{a}$ Etapa - Preparo das Técnicas de Mapeamento de Informação: essa etapa pretende atingir os seguintes objetivos: a) estabelecer critérios para reconhecer as fontes, serviços e sistemas compreendidos como recurso informacional relevante; b) identificar as dificuldades e debilidades da organização em relação a estas fontes, serviços e sistemas; c) determinar a natureza e as características desses recursos informacionais; d) avaliar, a partir dos elementos analisados, a política de informação da organização.

$4^{a}$ Etapa - Identificação dos Recursos de Informação da Organização: identifica os recursos de informação da organização. Também tem como propósito identificar as fortalezas e debilidades dos recursos. É importante mencionar que não se trata de um exercício de planejamento estratégico, cujo objetivo é identificar as fortalezas e as debilidades da organização; o objetivo é identificar os recursos.

Desse modo, de forma resumida, este método visa:

a) Mapear os fluxos de informação existentes nos diversos setores da organização;

b) Elaborar um fluxograma informacional para cada setor corporativo;

c) Agregar informações ao fluxograma inicial;

d) Elaborar um diagnóstico em relação aos fluxos informacionais e às pessoas diretamente $\mathrm{e}$ indiretamente ligadas aos referidos fluxos;

e) Identificar os tipos de informação utilizados no desenvolvimento das diferentes atividades;

f) Verificar as necessidades informacionais dos indivíduos nos diversos setores;

g) Identificar as tecnologias de informação e comunicação utilizadas para a gestão da informação;

h) Incentivar a cultura organizacional baseada em comprometimento e compartilhamento de informação e conhecimento;

i) Contextualizar os resultados visando a elaboração de uma política corporativa de gestão da informação.

\section{Proposta de um modelo}

A seguir será apresentada uma proposta de modelo de mapeamento de informação em ambiente corporativo, visando apoiar o desenvolvimento de atividades, bem como o processo de tomada de decisão. É importante mencionar que cada organização pode criar quantas categorias julgar necessárias para o mapeamento informacional, portanto as categorias que devem ser consideradas são variáveis, a partir da realidade de cada organização, ou seja, de suas especificidades, natureza, ramo, mercado, clientes, fornecedores, concorrentes etc. 
Outra questão importante que deve ser levada em conta, quando se elaborar as categorias para a realização do mapeamento informacional, é que as organizações, por sua própria natureza, produzem dados e informações diferenciados, assim como as demandas/necessidades de seus colaboradores são, também, específicas e, portanto, exigem gestões de informação diferenciadas. Nesse sentido, este modelo apenas sinaliza as várias possibilidades de aplicação do método Infomapping.

Para Burk Jr. e Horton Jr. (1988, p.116), as organizações precisam utilizar métodos e técnicas que identifiquem os recursos de informação existentes, assim como seus processos e fluxos. O método infomapping, conforme anteriormente mencionado, se apóia em quatro etapas básicas - 1) Mapeamento preliminar; 2) Determinação de custos e atribuição de valor; 3) Preparo das técnicas de mapeamento de informação; 4) Identificação dos recursos de informação da organização --, e seu resultado é extremamente benéfico para o tomador de decisão, visto que o método propicia maior segurança e melhor condição de análise para a resolução de problemas.

Para a definição das categorias de análise é preciso observar atentamente as características informacionais detectadas na segunda etapa (Determinação de custos e atribuição de valor), bem como as detectadas na quarta etapa (Identificação dos recursos de informação existentes na organização), porquanto é a partir dessas variáveis que cada organização poderá mapear o que é importante para o processo decisório no ambiente organizacional.

Burk Jr. e Horton Jr. (1988, p.117) explicam que conhecer os recursos informacionais permite a elaboração das categorias de análise realmente necessárias. Destacam as seguintes ações como fundamentais para a definição das categorias de análise:

- Identificar e reconhecer as fontes de informação relevantes que devem ser administradas como recursos de organização;

- Identificar os pontos fortes e fracos em relação a como a organização cria, adquire, armazena, usa, reutiliza e dissemina dado e informação;

- Identificar os custos e o valor de cada item de informação que faz parte da estrutura informacional da organização;

- Discriminar os custos de informação a partir das atividades e/ou tarefas apoiadas, por uso de cada departamento, por equipamento e meios de comunicação entre outras possibilidades;

- Determinar a natureza e as características gerais dos recursos de informação da organização;

- Determinar a qualidade, fidedignidade, pertinência etc.;

- Avaliar a adequação do recurso informacional à necessidade informacional da organização; 
- Avaliar se o uso dos recursos de informação agregou valor às ações informacionais da organização (BURK JR.; HORTON JR., 1988, p.117; tradução nossa).

A aplicação do infomapping requer um planejamento prévio, de forma que os envolvidos tenham clareza quanto aos objetivos a serem alcançados; visto que o método pode ser direcionado tanto para apenas um determinado problema informacional, como também, ao mesmo tempo, para diferentes problemas informacionais, tornando a aplicação mais complexa.

\subsection{Categorias de Análise das Atividades}

As categorias para a realização das análises informacionais das atividades/tarefas são vinculadas aos recursos informacionais existentes e às necessidades/demandas informacionais das pessoas que atuam na organização. A partir da aplicação do mapeamento, mais especificamente da etapa um (Mapeamento preliminar) e da etapa três (Preparo das técnicas de mapeamento de informação), é possível definir com objetividade quais devem ser as categorias de análise.

\begin{tabular}{|l|l|}
\hline \multicolumn{2}{|c|}{ Comunicação } \\
\hline 1 & Execuçäo Interna \\
\hline 2 & Contato com o Usuário \\
\hline 3 & Contato com Outras Seçốes \\
\hline 4 & Contato com Outros Setores Internos \\
\hline 5 & Contato com Org̣anizaçổes Externas \\
\hline 6 & Instâncias Superiores \\
\hline \multicolumn{2}{|c|}{ Obs.: Múltipla Escolha } \\
\hline
\end{tabular}

Figura 2 - Categoria Comunicação

Fonte - Elaborado pelas autoras

\begin{tabular}{|l|l|}
\hline \multicolumn{2}{|c|}{ Impacto Informacional } \\
\hline CTD & Contribui para a Tomada de Decisẫo \\
\hline PET & Produtividade na Execuçẫo de Tarefas \\
\hline OTE & Otimizaçẵo no Tempo de Execução \\
\hline MSE & Maior Segurança de Execuçäo \\
\hline NEl & Não Existe Impacto Informacional \\
\hline Obs:. MĹltipla Escolha \\
\hline
\end{tabular}

Figura 3 - Impacto Informacional.

Fonte - Elaborado pelas autoras

A categoria "Comunicação" é importante, na medida em que dimensiona a relação de uma determinada atividade com outros sujeitos/setores da organização.

Entende-se por comunicação organizacional o processo, através do qual, os indivíduos da organização obtêm as informações pertinentes sobre ela e as mudanças que nela ocorrem, desempenhando a função de fonte de informação para os indivíduos da organização (VALENTIM; ZWARETCH, 2007, p.45). 
Para as organizações, a comunicação é um elemento básico, pois através dela são formatadas as interações organizacionais entre as pessoas que nela atuam. A comunicação auxilia a promover mudanças que podem ser direcionadas tanto positivamente quanto negativamente.

Os fluxos informacionais ocorrem apoiados pela comunicação organizacional, por esse motivo a 'comunicação' foi escolhida como categoria.

A categoria "Impacto Informacional" é duplamente importante, porquanto é a partir dela que se verifica de que modo uma informação pode de fato subsidiar, de forma relevante, uma determinada atividade corporativa. O processo decisório depende essencialmente de informações relevantes para o negócio, de forma que o impacto informacional direcionará a gestão da informação.

O impacto da informação é uma categoria essencial, na medida em que proporciona uma avaliação correta dos fatores custo/valor, tão difícil de se observar ou medir em ambientes corporativos.

\begin{tabular}{|l|l|}
\hline \multicolumn{2}{|c|}{ Custo de Execução } \\
\hline ACE & Alto Custo de Execuçẫo \\
\hline MCE & Médio Custo de Execuçẫo \\
\hline BCE & Baixo Custo de Execuçâa \\
\hline
\end{tabular}

Figura 4 - Categoria - Custo de execução

Fonte - Elaborado pelas autoras

\begin{tabular}{|l|l|}
\hline \multicolumn{2}{|c|}{ Estratégico } \\
\hline AVE & Alto Valor Estratégico \\
\hline MVE & Médio Valor Estratégico \\
\hline BVE & Baixo Valor Estratégico \\
\hline
\end{tabular}

Figura 5 - Categoria - Estratégico

Fonte - Elaborado pelas autoras

Outra categoria extremamente importante para as organizações refere-se ao "Custo de Execução", ou seja, existem atividades corporativas que demandam um alto custo para serem executadas, outras, ao contrário, demandam um baixo custo. Essa variável, portanto, é importante para a gestão da informação, uma vez que sinaliza ao gestor quando o investimento informacional deve ser maior ou menor, justamente visando reduzir o alto custo de execução de uma determinada tarefa.

A categoria denominada de "Estratégico" indica o valor estratégico que uma informação possui para o desenvolvimento de uma atividade ou para a tomada de decisão relacionada ao negócio organizacional. Medir o valor de uma informação no âmbito corporativo é extremamente difícil, visto que o valor é subjetivo, porquanto o valor está vinculado ao uso que se dá para uma determinada informação, bem como ao resultado que se obtém desse uso. Aplicando o método Infomapping, é possível, a partir do levantamento de dados, analisar de forma mais segura o valor que uma informação tem para uma ação ou estratégia organizacional, visto que o resultado estará vinculado ao mapeamento de atividades desenvolvidas, 
sua seqüência, os atores participantes do processo, o(s) resultado(s) obtido(s) etc.

\begin{tabular}{|l|l|}
\hline \multicolumn{2}{|c|}{ Importância } \\
\hline $\mathrm{AGl}$ & Alto Grau de Importancia \\
\hline $\mathrm{MGl}$ & Médio Grau de Importância \\
\hline $\mathrm{BGl}$ & Baixo Grau de Importância \\
\hline
\end{tabular}

Figura 6 - Categoria - Importância

Fonte - Elaborado pelas autoras

\begin{tabular}{|l|l|}
\hline \multicolumn{2}{|c|}{ Tempo Despendido } \\
\hline ATD & Alto Tempo Despendido \\
\hline MTD & Médio Tempo Despendido \\
\hline BTD & Baixo Tempo Despendido \\
\hline
\end{tabular}

Figura 7 - Categoria - Tempo despendido

Fonte - Elaborado pelas autoras

Mapear o grau de importância de uma informação é essencial para a gestão da informação. Uma informação pode não ter um impacto significativo, assim como pode não ter um alto grau estratégico, mas pode ser fundamental para que um indivíduo desenvolva sua atividade cotidiana. Portanto, essa categoria precisa ser mapeada no contexto organizacional.

A categoria "Tempo Despendido" indica, para a gestão da informação, se uma informação auxilia ou não no que diz respeito ao tempo gasto por um indivíduo para a realização de uma determinada atividade. Muitas vezes uma atividade corporativa, que poderia ser facilmente desempenhada, demora por causa da dificuldade em acessar a informação no tempo certo.

\begin{tabular}{|l|l|}
\hline \multicolumn{2}{|c|}{$\begin{array}{c}\text { Redes de Relacionamentos - Institucional } \\
\text { Contatos Formais }\end{array}$} \\
\hline ACF & Alta Intensidade \\
\hline MCF & Média Intensidade \\
\hline BCF & Baixa Intensidade \\
\hline
\end{tabular}

Figura 8 - Categoria - Redes de relacionamento - Formal

Fonte - Elaborado pelas autoras

\begin{tabular}{|l|l|}
\hline \multicolumn{2}{|c|}{$\begin{array}{c}\text { Redes de Relacionamentos - Pessoal } \\
\text { Contatos Informais }\end{array}$} \\
\hline $\mathrm{ACl}$ & Alta Intensidade \\
\hline $\mathrm{MCl}$ & Média Intensidade \\
\hline $\mathrm{BCl}$ & Baixa Intensidade \\
\hline
\end{tabular}

Figura 9 - Categoria - Redes de relacionamento - Informal

Fonte - Elaborado pelas autoras

As redes de relacionamento são pertencentes aos fluxos informais da organização, e são vinculados ao âmbito da gestão do conhecimento. No entanto, é importante medir a intensidade tanto dos contatos formais, quanto dos contatos informais, uma vez que é dessa interação que, muitas vezes, resultam informações relevantes para a corporação. Uma vez explicitadas, passam a fazer parte dos fluxos formais, ou seja, vinculados ao âmbito da gestão da informação. Por isso, mapear essas 
redes é, também, fundamental para a gestão da informação, visto que sinalizará os líderes corporativos, bem como indicará o nível de produção de conhecimento (tácito) e informação (explícito).

\begin{tabular}{|l|l|}
\hline \multicolumn{2}{|c|}{ Automação de Sistemas } \\
\hline ATA & Atividade Totalmente Automatizada \\
\hline APA & Atividade Parcialmente Automatizada \\
\hline ANA & Atividade Nä́ Automatizada \\
\hline ANN & Atividade Năo Necessita de Automaçăo \\
\hline
\end{tabular}

Figura 10 - Categoria - Automação de Sistemas

Fonte - Elaborado pelas autoras

\begin{tabular}{|l|l|}
\hline \multicolumn{2}{|c|}{ Acesso a Informação } \\
para Desenvolver a Atividade \\
\hline AGF & Alto Grau de Facilidade \\
\hline MGF & Médio Grau de Facilidade \\
\hline BGF & Baixo Grau de Atividade \\
\hline
\end{tabular}

Figura 11 - Categoria - Acesso à informação

Fonte - Elaborado pelas autoras

A categoria "Automação de Sistemas" visa sinalizar quais atividades estão automatizadas, isto é, quais atividades utilizam tecnologias de informação e comunicação, visando otimizar os fluxos e processos corporativos. Esse mapeamento ajudará a gestão da informação a integrar seus diferentes sistemas ao sistema da atividade em questão, de forma a melhorar a geração, o compartilhamento, a disseminação e o uso de informações.

Outra categoria importante, "Acesso à Informação para Desenvolver a Atividade", subsidiará a gestão da informação quanto à facilidade que os indivíduos atuantes na organização têm para acessar uma determinada informação necessária para o desenvolvimento de uma atividade corporativa.

\begin{tabular}{|l|l|}
\hline \multicolumn{2}{|c|}{ Capacitação para Desenvolver a Atividade } \\
\hline $\mathrm{AGC}$ & Alto Grau de Capacitaçâa \\
\hline MGC & Médio Grau de Capacitaçăo \\
\hline $\mathrm{BGC}$ & Baixo Grau de Capacitaçẵo \\
\hline
\end{tabular}

Figura 12 - Categoria - Capacitação para Desenvolver a Atividade.

Fonte - Elaborado pelas autoras.

\begin{tabular}{|l|l|}
\hline \multicolumn{2}{|c|}{ Nivel de Dificuldade de Execução } \\
\hline AND & Alto Nivel de Dificuldade \\
\hline MND & Médio Nivel de Dificuldade \\
\hline BND & Baixo Nivel de Dificuldade \\
\hline
\end{tabular}

Figura 13 - Categoria - Nível de Dificuldade de Execução.

Fonte - Elaborado pelas autoras.

A categoria "Capacitação para Desenvolver a Atividade" dimensiona corretamente o tipo, o nível, a quantidade e a forma em que uma informação deve ser processada e disseminada. A gestão da informação poderá ajustá-la, quantas vezes forem necessárias, de forma que o usuário da informação não seja impedido de usá-la por não compreender a informação ou não vinculá-la à resolução de um problema corporativo. 
O nível de dificuldade de execução é a categoria que dimensiona o grau de dificuldade de execução de uma atividade corporativa ou de um processo decisório. Quanto maior o grau de dificuldade, maior será a responsabilidade da gestão da informação, visto que deve encontrar recursos informacionais que diminuam esse grau de dificuldade.

\begin{tabular}{|l|c|c|c|c|}
\hline $\begin{array}{c}\text { Número da } \\
\text { Atividade }\end{array}$ & $\begin{array}{c}\text { Grau } \\
\text { Estratégico }\end{array}$ & $\begin{array}{c}\text { Grau de } \\
\text { Importância }\end{array}$ & $\begin{array}{c}\text { Tempo } \\
\text { Despendido }\end{array}$ & Observações \\
\hline & & & & \\
\hline & & & & \\
\hline & & & & \\
\hline & & & & \\
\hline
\end{tabular}

$A=$ Alto; M=Médio; $B=B a i x o$

Figura 14 - Planilha Geral.

Fonte - Elaborado pelas autoras.

Após o mapeamento de cada atividade de um determinado setor, é importante elaborar uma planilha geral, relacionando cada atividade e o mapeamento quanto às variáveis (categorias) previamente estabelecidas. Essa planilha geral fornecerá todos os elementos de análise para as pessoas que atuam na gestão da informação. A partir da análise do mapeamento, estabelece-se um plano de ação informacional ou uma política de informação corporativa, visando maior eficiência do negócio organizacional.

Esse método auxilia enormemente a gestão da informação em espaços organizacionais, visto que a informação se constitui em um bem estratégico. Para Burk Jr e Horton Jr. (1988, p.178), os recursos informacionais, quando bem geridos, promovem o alcance dos objetivos organizacionais de forma mais eficiente. Logicamente, essa ação requer o uso de tecnologias de informação e comunicação, ferramentas essenciais para a gestão da informação, principalmente quando se aplica o infomapping.

\section{Considerações finais}

O método Infomapping criado por Burk Jr. e Horton Jr. é sem dúvida um instrumento para a gestão da informação eficiente no contexto organizacional. As possibilidades de mapeamento e análise dos fluxos formais existentes nesse ambiente são muito amplas. Portanto, cada organização deve estabelecer o seu próprio rol de categorias de análise visando conhecer todas as variáveis para o desenvolvimento de atividades, bem como compreender melhor o processo de tomada de decisão.

A gestão da informação pode utilizar diferentes métodos e técnicas que, aplicadas isoladamente ou em conjunto, obterão resultados efetivos 
quanto à geração, uso e compartilhamento de dados, informação e conhecimento. O método Infomapping propicia, aos profissionais que atuam diretamente na gestão da informação, uma visão ampla sobre o contexto informacional do ambiente corporativo, trazendo maior efetividade ao trabalho desenvolvido por eles.

\section{Referências}

CAPRA, F. O ponto de mutação. 28 ed. São Paulo: Cultrix, 2002. 447p.

BOLÍVAR, H.; CASTRO, A. M. G.; HERNÁNDEZ SANCHES, A.; OVELAR AGUILERA, M. G.; SARMIENTO, E. M. A. Sistemas de información gerencial (SIG). Quito: Isnar, 1997.

BURK JR., C. F.; HORTON, F. W. Infomap: a complete guide to discovering corporate information resources. New York: Englewood Cliffs/Prentice Hall, 1988. 233p.

CASTRO, A. M. G. de; LiMA, S. M. V. L.; CARVALHO, J. R. P. de. Planejamento de C\&T: sistema de informação gerencial. Brasília: Embrapa, 1999. 328p.

PONJUÁN DANTE, G. Gestión de información en las organizaciones: principios, conceptos y aplicaciones. Santiago: CECAPI, 1998. 222p.

VALENTIM, M. L. P.; ZWARETCH, N. S. Comunicação organizacional/comunicação informacional no processo de inteligência competitiva organizacional. In: VALENTIM, M. L. P. (Org.). Informação, conhecimento e inteligência organizacional. 2.ed. Marília: FUNDEPE, 2007. 278p. p.45-59

VALENTIM, M. L. P. (Org.). Informação, conhecimento e inteligência organizacional. 2.ed. Marília: FUNDEPE, 2007. 278p. 